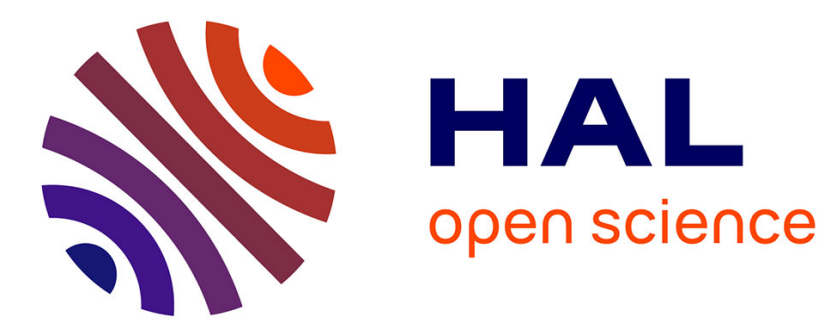

\title{
Modelisation d'une torche à courant continu
}

J.-M. Bauchire, Jean Jacques Gonzalez, A. Gleizes

\section{To cite this version:}

J.-M. Bauchire, Jean Jacques Gonzalez, A. Gleizes. Modelisation d'une torche à courant continu. Journal de Physique III, 1997, 7 (4), pp.829-837. 10.1051/jp3:1997158 . jpa-00249616

\section{HAL Id: jpa-00249616 https://hal.science/jpa-00249616}

Submitted on 1 Jan 1997

HAL is a multi-disciplinary open access archive for the deposit and dissemination of scientific research documents, whether they are published or not. The documents may come from teaching and research institutions in France or abroad, or from public or private research centers.
L'archive ouverte pluridisciplinaire HAL, est destinée au dépôt et à la diffusion de documents scientifiques de niveau recherche, publiés ou non, émanant des établissements d'enseignement et de recherche français ou étrangers, des laboratoires publics ou privés. 


\title{
Modélisation d'une torche à courant continu
}

\author{
J.-M. Bauchire, J.-J. Gonzalez (*) et A. Gleizes \\ Centre de Physique des Plasmas et de leurs Applications de Toulouse (**), \\ Université Paul Sabatier, 118 route de Narbonne, 31062 Toulouse Cedex 4, France
}

(Reçu le 24 mai 1996, révisé le 6 janvier 1997, accepté le 9 Janvier 1997)

PACS.52 35.Ra - Plasma turbulence

PACS.52.65.-y - Plasma simulation

PACS $5275 \mathrm{Hn}$ - Plasma torches

Résumé. - Nous présentons dans cet artıcle la modélisation numérıque à deux dimensions d'une torche à plasma d'argon à courant continu. Ce modèle, basé sur la résolution des équatıons de Navier-Stokes et de l'électromagnétisme par la méthode des volumes de contrôle de Patankar, permet de décrire les champs de vitesse et de température dans la zone inter-électrodes et dans le jet de plasma. La turbulence est prise en compte à travers un modèle de longueur de mélange de Prandtl Les résultats présentés font apparaître l'influence de la turbulence sur les champs d'écoulement ainsı que l'influence de l'intensité du courant d'arc et du débit de gaz injecté sur les caractéristıques du plasma

\begin{abstract}
We present in this paper a 2D numerical modelling of a dc argon plasma torch. This model is based on the resolution of the electromagnetism and Navier-Stokes equations by the control volume method of Patankar. This model allows to describe the velocity and temperature fields between the electrodes and in the plasma jet. Turbulence phenomenon is taken into account by a Prandtl's mixing length model The results show the influence of turbulence on the flow fields and also the influence of current intensity and inlet mass flow rate on the characteristics of the plasma.
\end{abstract}

\section{Introduction}

L'utilisation d'arcs électriques pour obtenir des gaz à très hautes températures, à fort rayonnement et la présence d'espèces ionisées et excitées, offrent de nombreuses applications industrielles qui font l'objet de développements importants. Parmi celles-ci, la torche à plasma, représentée par une gamme technologique très diversifiée suscite un intérêt croissant. Elle permet notamment de traiter des problèmes de résistance à l'usure et à la corrosion mais aussi d'isolation thermique et électrique.

Dans sa version la plus simple, la torche est composée de deux électrodes coaxıales refroidies. Au centre, la cathode, généralement en tungstène thorié, et autour, faisant office de tuyère, l'anode en cuivre. Le gaz, injecté parallèlement à l'axe entre les deux électrodes traverse l'arc

$\left(^{*}\right)$ Auteur auquel dolt être adressée la correspondance (e-mail : jjg@cpa11 ups-tlse.fr)

$\left({ }^{* *}\right)$ E.S.A $n^{\circ} 5002$

(C) Les Éditions de Physique 1997 
électrique formant ainsi un jet de plasma. La complexité du système peut varier suivant la forme de la tuyère ou le mode de stabilisation de l'arc, mais même dans les configurations les plus simples, une utilisation efficace de ce jet nécessite un bonne connaissance de l'influence des paramètres de fonctionnement de la torche.

Le but de notre travail a été de développer un modèle mathématique et numérique à deux dimensions afin de prédire les champs de vitesse et de température dans le jet de plasma mais aussi dans la zone inter-électrodes d'une torche à courant continu.

\section{Modèle mathématique}

2.1. HypothèSES ET ÉquATIONS GÉNÉRALES. - Ce modèle est basé sur les hypothèses suivantes : le système est à symétrie cylindrique, l'écoulement est stationnaire et les effets de la gravité sont négligés. Le plasma est en Équilibre Thermodynamique Local (ÉTL) et le rayonnement est traité à travers le coefficient d'émission nette. On suppose également la pression égale à une atmosphère.

À partir de ces hypothèses, on obtient le système suivant composé par les équations de Navier-Stokes et de l'électromagnétisme :

Conservation de la masse .

$$
\frac{\partial}{\partial x}(\rho u)+\frac{1}{r} \frac{\partial}{\partial r}(\rho r v)=0 .
$$

Conservation de la quantité de mouvement axiale :

$$
\rho u \frac{\partial u}{\partial x}+\rho v \frac{\partial u}{\partial r}=-\frac{\partial p}{\partial x}+2 \frac{\partial}{\partial x}\left(\mu_{\mathrm{e}} \frac{\partial u}{\partial x}\right)+\frac{1}{r} \frac{\partial}{\partial r}\left(\mu_{\mathrm{e}} r \frac{\partial u}{\partial r}\right)+\frac{1}{r} \frac{\partial}{\partial r}\left(\mu_{\mathrm{e}} r \frac{\partial v}{\partial x}\right)+\jmath_{r} B_{\theta} .
$$

Conservation de la quantité de mouvement radiale :

$$
\rho u \frac{\partial v}{\partial x}+\rho v \frac{\partial v}{\partial r}=-\frac{\partial p}{\partial r}+\frac{\partial}{\partial x}\left(\mu_{\mathrm{e}} \frac{\partial v}{\partial x}\right)+\frac{2}{r} \frac{\partial}{\partial r}\left(\mu_{\mathrm{e}} r \frac{\partial v}{\partial r}\right)+\frac{\partial}{\partial x}\left(\mu_{\mathrm{e}} \frac{\partial u}{\partial r}\right)-\frac{2 \mu_{\mathrm{e}} v}{r^{2}}-j_{x} B_{\theta} .
$$

Conservation de l'énergie :

$\rho u \frac{\partial h}{\partial x}+\rho v \frac{\partial h}{\partial r}=\frac{\partial}{\partial x}\left(\Gamma_{\mathrm{T}} \frac{\partial h}{\partial x}\right)+\frac{1}{r} \frac{\partial}{\partial r}\left(r \Gamma_{\mathrm{T}} \frac{\partial h}{\partial r}\right)+\frac{j_{x}^{2}+j_{r}^{2}}{\sigma}-4 \pi \varepsilon_{\mathrm{N}}+\frac{5}{2} \frac{k_{\mathrm{B}}}{e}\left(\frac{j_{x}}{C_{\mathrm{p}}} \frac{\partial h}{\partial x}+\frac{j_{r}}{C_{\mathrm{p}}} \frac{\partial h}{\partial r}\right)$

avec

$$
\Gamma_{\mathrm{T}}=\frac{\kappa}{C_{\mathrm{p}}}+\frac{\mu_{\mathrm{t}}}{\operatorname{Pr}_{\mathrm{T}}}
$$

Conservation du courant :

$$
\frac{\partial j_{x}}{\partial x}+\frac{1}{r} \frac{\partial}{\partial r}\left(r j_{\mathbf{r}}\right)=0
$$

où $u$ et $v$ sont les composantes axiale et radiale de la vitesse, $\rho$ est la densité de masse, $x$ et $r$ représentent les variables spatiales dans les directions axiale et radiale respectivement. $p$ est la pression, $h$ l'enthalpie spécifique, $\mu_{\mathrm{e}}$ la viscosité effective et $\mu_{\mathrm{t}}$ la viscosité turbulente. $C_{\mathrm{p}}$, $\kappa$, et $\sigma$ sont la chaleur spécifique et les conductivités thermique et électrique. $\jmath_{x}$ et $\jmath_{r}$ sont les composantes axiale et radiale de la densité de courant, $k_{\mathrm{B}}$ la constante de Boltzmann, $e$ la charge élémentaire de l'électron, $\operatorname{Pr}_{\mathrm{T}}$ le nombre de Prandtl et $\varepsilon_{\mathrm{N}}$ le coefficient d'émission nette. 


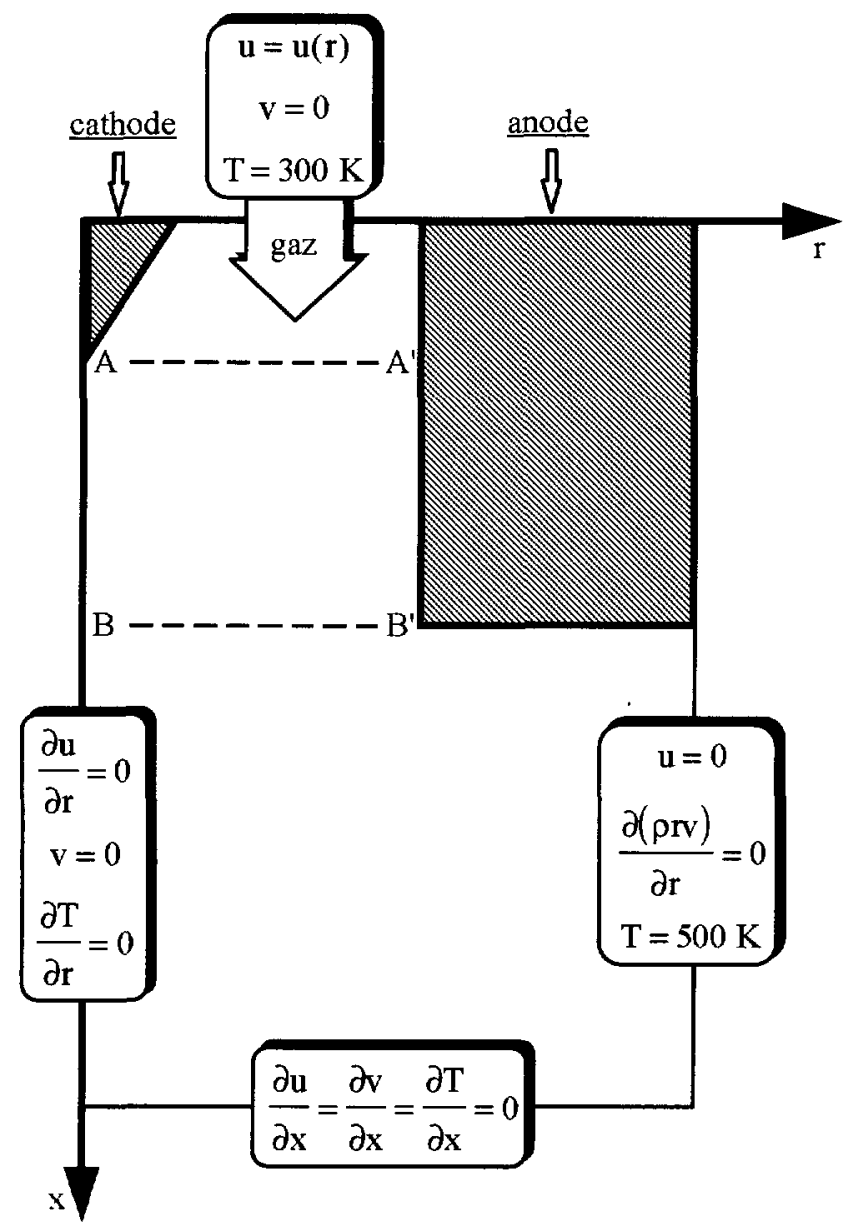

Fig. 1. - Domaine de calcul et conditions aux limites.

[Computation domain and boundary conditions.]

La composante azimutale de l'induction magnétique est telle que :

$$
B_{\theta}=\frac{\mu_{0}}{r} \int_{0}^{r} j_{x} \xi \mathrm{d} \xi
$$

où $\mu_{0}$ est la perméabilité du vide. La résolution de ces équations de conservation est assurée par la méthode des volumes de contrôle de Patankar [1].

2.2. Modèle DE TURBUlenCE. - Le phénomène de turbulence est pris en compte à travers la viscosité effective qui est la somme des viscosités laminaire et turbulente. Différents modèles tels que longueur de mélange ou $k-\varepsilon$ ont été utilisés [2]. Dans cet article nous ne présentons que le modèle de Prandtl. La viscosité turbulente est obtenue suivant cette hypothèse par :

$$
\mu_{\mathrm{t}}=\rho l_{\mathrm{m}}^{2}\left|\frac{\partial u}{\partial r}\right|
$$




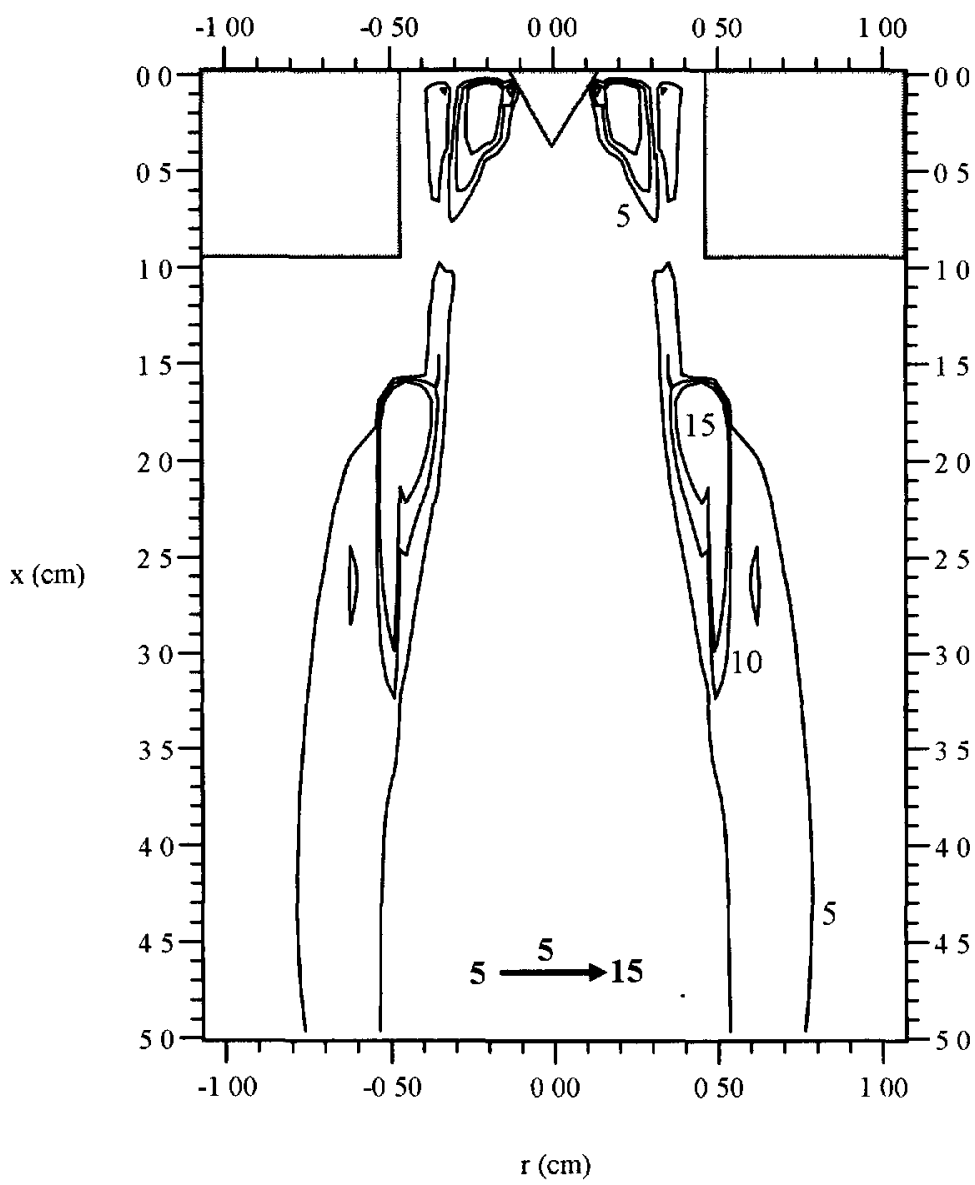

Fig. 2. - Isocontours du nombre de Reynolds local turbulent

[Contours of the turbulent local Reynolds number]

À partir des travaux de Nicolet et al. [3], nous obtenons pour la zone gainée du plasma, les valeurs de la longueur de mélange $\left(l_{\mathrm{m}}\right)$ et du nombre de Prandtl $\left(\operatorname{Pr}_{\mathrm{T}}\right)$ suivantes :

$$
\begin{aligned}
& \text { pour } y \leq y_{\mathrm{c}}, \quad l_{\mathrm{m}}=0,4 y\left[1-\exp \left(\frac{-y \sqrt{\rho \mu^{*}\left|\frac{\partial u}{\partial r}\right|^{*}}}{26 \mu^{*}}\right)\right], \\
& \text { pour } \quad y_{\mathrm{c}} \leq y \leq R, \quad l_{\mathrm{m}}=0,075 R, \\
& \text { pour } \quad y \geq 0,05 R, \quad \operatorname{Pr}_{\mathrm{T}}=0,95-0,45\left(\frac{y}{R}\right)^{2}, \\
& \text { pour } y<0,05 R, \quad \operatorname{Pr}_{\mathrm{T}}=3-\frac{20 y}{R}(3-0,949),
\end{aligned}
$$

où $R$ est le rayon de la tuyère, $y$ est la distance à partir de la paroi de l'anode telle que $y=R-r+r_{\mathrm{g}} \cdot r_{\mathrm{g}}$ est la rugosité de paroi égale à $0,01 \mathrm{~mm}$ et $y_{\mathrm{c}}$ est obtenu par continuité de $l_{\mathrm{m}}$ entre les équations (8) et (9) Les valeurs $\left(^{*}\right)$ sont prises à la paroi. Dans la zone de jet libre, on utilise les expressions de Gonzalez [4] : la longueur de mélange est déduite de l'équation (9), le nombre de Prandtl est obtenu par l'équation (10) si $r<R$ et par $\operatorname{Pr}_{\mathbf{T}}=0,95$ si $r \geq R$. 


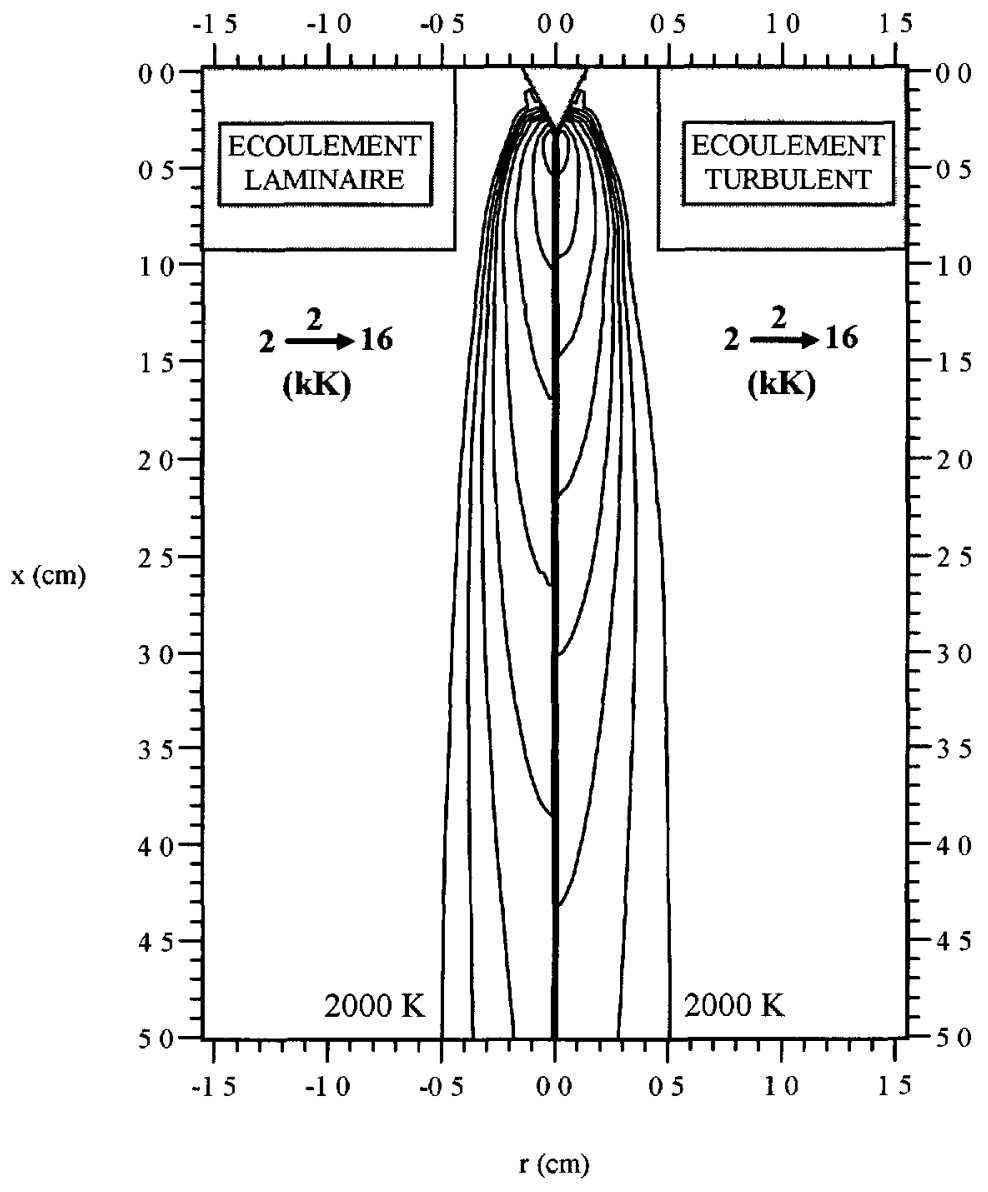

F1g. 3 - Isothermes du plasma en écoulement laminaire et turbulent.

[Temperature contours of the plasma for laminar and turbulent flows.]

2.3. Domaine De discrétisation et Conditions aux limites. - Le domaine de calcul et les conditions aux limites sont portés sur la figure 1. La densité de courant axial est imposée le long de la ligne AA' suivant une fonction exponentielle d'après Hsu et al. [5]. Sur la ligne BB', le potentiel électrique est supposé nul, matérialisant une anode fictive poreuse. L'attachement du pied d'arc sur l'anode réelle n'est donc pas modélisé. Le profil de vitesse $u(r)$ d'arrivée de gaz est parabolique. Le gaz plasmagène considéré est l'argon dont les propriétés thermodynamiques et les coefficients de transport sont issus de Mostaghimi [6]

La torche possède une tuyère de $9,2 \mathrm{~mm}$ de long et un diamètre de $9,4 \mathrm{~mm}$. La cathode mesure 2,7 mm de long pour 1,3 mm de large. Les calculs sont effectués pour un domaine de discrétisation d'une longueur de $50 \mathrm{~mm}$ et une largeur de $15 \mathrm{~mm}$, soit une grille de $85 \times 35$ points. Des calculs préliminaires ont été fait sur l'influence des dimensions radrale et axiale du domaine de calcul. Dans cette étude nous avons comparé les résultats obtenus avec des maillages de $85 \times 35$ points (axialement $\times$ radialement), $167 \times 35$ points $(10 \mathrm{~cm}$ par $1,5 \mathrm{~cm}$ ) et $85 \times 42(5 \mathrm{~cm}$ par $3 \mathrm{~cm})$. La bonne stabilité et la concordance des résultats obtenus à partir des différentes grilles nous a conduit à utiliser la grılle $85 \times 35$ qui réalıse le meilleur compromis 


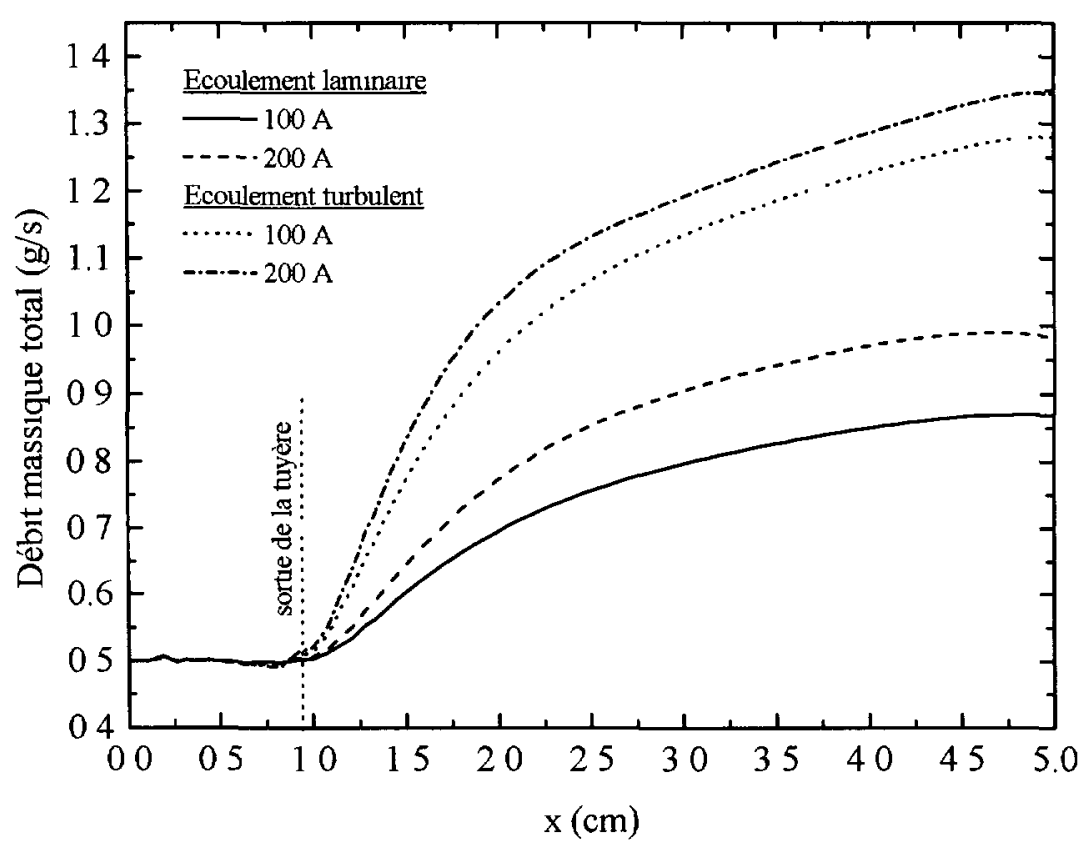

Fig. 4. - Évolution du débit massique total suvvant l'axe de la décharge.

[Total mass flow rate versus the axial position.]

temps-précision. Les champs de vitesse et de température ont été calculés pour des intensités comprises entre 100 et $200 \mathrm{~A}$ et pour des débits d'entrée de gaz compris entre 0,5 et $1,0 \mathrm{~g} \mathrm{~s}^{-1}$.

\section{Résultats}

3.1. Influence de la turbulence. - Dans un écoulement laminaire les forces d'inertie axiales sont négligeables devant les forces de viscosité. Ceci est rarement le cas pour les jets émergeant d'un orifice dans un milieu infini Nous avons tracé sur la figure 2 les iso-contours du nombre de Reynolds local turbulent $\operatorname{Re}_{\mathrm{T}}=\frac{\mu_{\mathrm{t}}}{\mu}[7]$. Ils font apparaître une zone de mélange c'est-à-dire une couche limite entre le jet et le gaz ambiant. Dans cette zone frontière du jet, le transfert de matière n'est pas unıquement dû à la diffusion mais aussi aux tourbillons turbulents qui créent un pompage du gaz froid environnant vers le centre du jet.

Nous avons tracé sur la figure 3 , pour une intensité de $100 \mathrm{~A}$ et un débit initial de $0,5 \mathrm{~g} \mathrm{~s}^{-1}$, les isothermes du plasma pour un écoulement laminaire à gauche et pour un écoulement turbulent à droite. Nous avons aussi tracé sur la figure 4 l'évolution du débit massique total pour des intensités de 100 et $200 \mathrm{~A}$.

On peut observer deux conséquences du phénomène de pompage décrit plus haut, à savoir un refroidissement de la colonne de plasma (Fig. 3) et une augmentation du débit massique liée également à l'entraînement par frottement visqueux (Fig. 4).

3.2. Influence de L'Intensité. - Sur la figure 5 sont portées les isothermes du plasma pour des intensités de courant d'arc de 100 et $200 \mathrm{~A}$ et un débit initial d'argon de $0,5 \mathrm{~g} \mathrm{~s}^{-1}$. On peut observer sur cette figure une élévation de la température avec l'intensité $I$. 


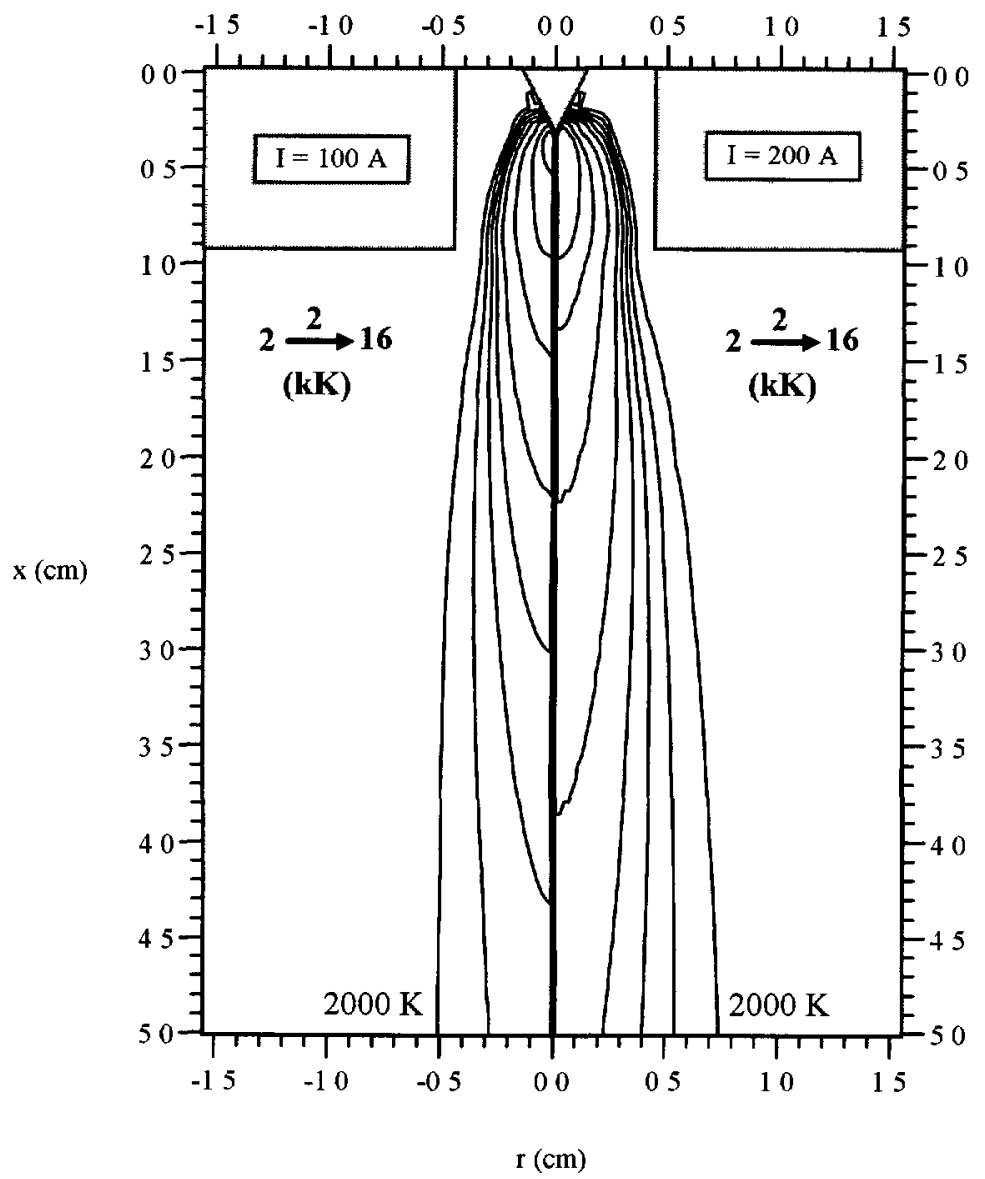

Fig 5 - Isothermes du plasma pour des intensités de courant d'arc de $100 \mathrm{~A}$ et $200 \mathrm{~A}$ [Temperature contours of the plasma for current intensities of $100 \mathrm{~A}$ and $200 \mathrm{~A}$.]

Tableau I. - Purssance effectıve dans le plasma.

[Power in the plasma.]

\begin{tabular}{|c|c|c|}
\hline Intensité (A) & 100 & 200 \\
\hline Puissance (W) & 1200 & 1500 \\
\hline
\end{tabular}

Ce phénomène est lié à l'augmentation de la puissance fournie au plasma avec l'intensité (Tab. I).

Mais on remarque également sur la figure 4 qu'une hausse de l'intensité conduit à une augmentation du débit. La température étant plus élevée dans la tuyère (pour $200 \mathrm{~A}$ ), la densité de masse est plus faible, il s'en suit une augmentation de la vitesse axiale afin d'assurer la conservation du débit $D: D=2 \pi \int_{0}^{R} \rho u r \mathrm{~d} r$. Les forces de viscosité importantes et la vitesse du jet à la sortie plus élevée conduisent à un entraînement du gaz ambiant plus grand et donc à cette augmentation du débit dans la zone non gainée. 


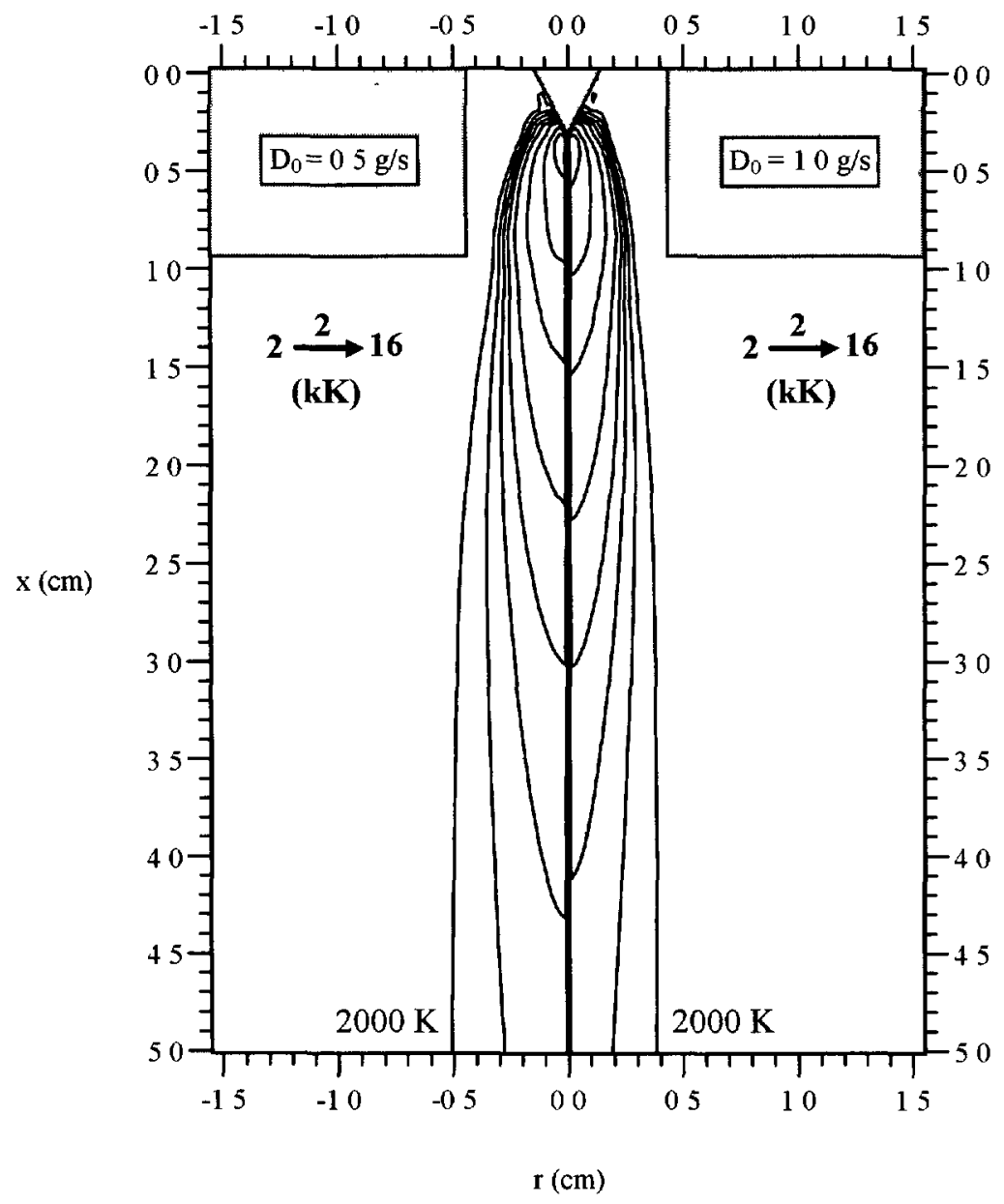

Fig. 6. - Isothermes du plasma pour des débits d'entrée d'argon de $0,5 \mathrm{~g} \mathrm{~s}^{-1}$ et $1,0 \mathrm{~g} \mathrm{~s}^{-1}$.

[Temperature contours of the plasma for an inlet mass flow rate of 0.5 and $10 \mathrm{~g} \mathrm{~s}^{-1}$.]

3.3. InfluenCE DU DÉBit DE GAZ INJECTÉ. - La figure 6 met en évidence l'évolution des ssothermes du plasma en fonction du débit d'argon initial pour une intensité de $100 \mathrm{~A}$ et un écoulement turbulent. On peut observer qu'une hausse du débit de gaz initial conduit à une contraction et à un réchauffement de la colonne de plasma.

En effet, cette augmentation du débit entraîne une augmentation des pertes (par convection, turbulence) et donc conduit à une élévation du champ électrique moyen. Donc à intensité constante, la conductance diminue c'est-à-dire que le rayon de conduction du plasma diminue quand le débit initial augmente. Ceci entraîne la contraction de la colonne et de ce fait l'élévation de la température sur l'axe.

\section{Conclusion}

Bien que la modélisation des torches à plasma se heurte à des problèmes liés aux phénomènes aux électrodes, aux interactions arc-écoulement ou même à un manque de données sur les 
propriétés thermodynamıque et de transport, les résultats obtenus lors de cette première étude montrent un bon accord avec les résultats de la littérature.

On a pu mettre en évidence l'importance des paramètres de fonctionnement sur les caractéristiques du plasma mass aussi mesurer l'influence de la turbulence sur les propriétés de l'écoulement.

Ce modèle va nous permettre une étude de procédés plus complexes comme la projection de particules ainsi qu'une comparaison avec des résultats expérimentaux.

\section{Remerciements}

Les auteurs remercient le Conseil Régional Midi-Pyrénées pour son aide financière dans cette étude avec le projet de recherche RECH/9300395.

\section{Bibliographie}

[1] Patankar S.V., Numerical Heat Transfer and Fluid Flow (McGraw-Hill, 1980).

[2] Bauchire J.M., Gonzalez J J and Gleizes A., A physical modelling of a dc plasma torch, Proc. of the $12^{\text {th }}$ International Symposium on Plasma Chemistry, vol. 3 (1995) pp. 17611766.

[3] Nicolet W.E. et al., Analytical and design study for a high-pressure, high-enthalpy constricted arc heater, Acurex Corporation, AEDC, TR.75.47 (1975).

[4] Gonzalez J.J., Modélisation d'un arc transféré de forte puissance (1 MW), Thèse de Doctorat (Université Paul Sabatier, $n^{\circ} 1100,1992$ ).

[5] Hsu K.C., Etemadi K. and Pfender E., Study of the free-burning high intensity argon arc, J. Appl. Phys. 54 (1983) 1293-1301.

[6] Mostaghimi J., The effects of low ionization potential contaminants on the transport properties of thermal plasmas, Ph. D. Thesis (University of Minnesota, USA, 1982).

[7] Rodi W, Turbulence models and their applications, Eyrolles Éds., ISSN 0399-4198, vol. 2 (1984). 